\title{
Schizophrenia genetics moves into the light
}

\author{
David Curtis
}

\section{Summary}

Two recent reports convincingly demonstrate that the risk of schizophrenia is influenced by common variation of $\mathrm{C4}$, which codes for complement component 4 , and by rare, disruptive mutations of SETD1A, which codes for a histone methylase. These important results open the way for major advances in our understanding of the fundamental biological processes leading to disease.

\section{Declaration of interest}

None.

\section{Copyright and usage}

(c) The Royal College of Psychiatrists 2016.
David Curtis is an Honorary Professor at the UCL Genetics Institute and also at the Centre for Psychiatry, Barts and the London School of Medicine and Dentistry. He carries out research in psychiatric and statistical genetics.

Recent months have seen dramatic advances in schizophrenia genetics research, with the first definitive identification of specific coding variations directly affecting schizophrenia risk. Two separate genes have been implicated: common variation in C4, which codes for complement component 4 , exerts a modest effect on schizophrenia risk that, in the large samples studied, ${ }^{1}$ was statistically significant at $P=3.5 \times 10^{-24}$; by contrast, very rare variants disrupting SETD1A, which codes for a component of a histone methyl transferase, exert a very large effect on risk, ${ }^{2}$ which was significant at $P=3.3 \times 10^{-9}$. The importance of these findings does not relate to their immediate clinical implications. At an individual level, $C 4$ variation has a negligible effect on risk and only a very small fraction of patients will possess SETD1A variants. Rather, these findings for the first time give us a concrete opening into understanding the biology of schizophrenia.

Previous findings in schizophrenia genetics could be broadly grouped into three main classes: individual variants that were of questionable statistical significance and which await replication; genetic markers that showed statistically robust association but which were not themselves causative and that did not implicate any individual gene; ${ }^{3}$ rare chromosomal deletions and duplications that definitely exerted a large effect on risk but which each contained multiple genes, meaning that one could not determine which were of relevance. ${ }^{4}$ Arguably, the single exception to this would be that some pathogenic chromosome 2 deletions are so small as to only affect a single gene, NRXN1. Another exception could be made for $D I S C 1$, in that the originally reported translocation does definitely increase the risk of schizophrenia and other mental disorders. ${ }^{5}$ However, no other disease-causing variants in DISC1 have been conclusively identified, despite extensive attempts. Thus, the latest findings are widely perceived as marking a real step forward and as taking us into new territory.

\section{Findings relating to $C 4$}

The study of $C 4$ was driven by the finding that many markers in the HLA region, where it is located, demonstrate significant association with schizophrenia. ${ }^{1}$ Intensive studies of the gene revealed that it could vary in any of three different ways: the number of copies of the gene on each chromosome can vary; each copy can be 'long' or 'short', depending on whether or not it harbours a small insertion of viral origin; a variation in DNA sequence means that each copy can be denoted as either ' $C 4 A$ ' or ' $C 4 B$ ', with these two forms of the gene producing versions of the protein product having different properties. The specific C4 gene or genes found on a chromosome can be referred to as a haplotype and the study showed that four different haplotypes commonly occur. The level of $C 4 A$ expression produced by each haplotype varies and the predicted $C 4 A$ expression correlated with schizophrenia risk at $P=3.5 \times 10^{-24}$. The highest risk haplotype, consisting of two long forms of $C 4 A$, confers increased risk by a factor of 1.27 over the lowest risk haplotype, consisting of a single copy of the short form of $C 4 B$. The study also demonstrated that complement component 4 is found on neurons and synapses, so it would be legitimate to speculate that increased $C 4 A$ expression could lead to increased synaptic pruning. Indeed, the authors were able to demonstrate that mice manipulated to lack $C 4$ had reduced synaptic pruning.

\section{Findings relating to SETD1A}

The SETD1A study represented an extension and confirmation of previous research that had demonstrated that people with schizophrenia with unaffected parents could possess de novo variants, i.e. not found in either parent, which disrupted SETD1A. ${ }^{6}$ The recent study demonstrated this was a more widespread phenomenon, confirming its validity. ${ }^{2}$ Loss of function (LOF) variants were defined as those which would seriously disrupt correct translation of the gene, either through introducing a premature stop codon, shifting the reading frame or preventing normal splicing. Such LOF variants were found in a total of 10 out of nearly 8000 people with schizophrenia but none of over 13000 controls. This implies that if one has only one working copy of this gene, rather than two, then the risk of developing schizophrenia is very substantially increased. LOF variants in SETD1A were also identified in 4 out of 3148 children with diverse, severe, developmental disorders, suggesting that this can be an alternative outcome. SETD1A is involved in the methylation of a lysine residue in histone tails, a process that leads to increased expression of other genes. In particular, there are suggestions that this may affect genes involved in synaptic 
function and GABAergic signalling, as well as dopaminergic and glutamatergic transmission. ${ }^{7}$ It is of note that mutations in genes with similar function to SETD1A are known to cause developmental disorders such as Wiedemann-Steiner syndrome, Kleefstra syndrome and Kabuki syndrome. Likewise, there is independent evidence that other genes involved in histone modification also influence risk of schizophrenia. ${ }^{8,9}$

\section{Implications of recent findings}

Thus, these two findings appear to implicate two very different biological pathways affecting schizophrenia risk. The former demonstrates a role for complement, perhaps specifically that increased $C 4 A$ expression leads to increased synaptic pruning. Although the effect of naturally occurring variation is small, this does not mean that pharmacological targeting of this process might not exert substantial effects on the development and/or course of schizophrenia. The latter finding implicates broad effects on gene expression that seem to result in a surprisingly narrow phenotypic effect. Because of the magnitude of this effect, one could argue that testing for SETD1A variants should become part of the routine management of schizophrenia, alongside testing for pathogenic copy number variants. ${ }^{4}$

The implications of these findings are so far-reaching that it is impossible to do them justice in the space available. Both will be followed by molecular studies that will seek to gain further insight into the mechanisms by which these variants affect risk and this process is likely to lead to the implication of additional related genes. Thus, although the contribution which the currently identified variants make towards the overall risk of schizophrenia in the population is small, the concrete nature of these findings means that it is reasonable to expect that they will progress towards a situation in which we attain a far more substantial understanding of the molecular mechanisms involved in schizophrenia aetiology. Many workers in psychiatric genetics feel that the field has entered a long-awaited new phase and look forward with enthusiasm to further developments.

David Curtis, MD, PhD, FRCPsych, UCL Genetics Institute, UCL, and Centre for Psychiatry, Barts and the London School of Medicine and Dentistry, Darwin Building, Gower Street, London WC1E 6BT, UK. Email: d.curtis@ucl.ac.uk

First received 22 Mar 2016, accepted 5 May 2016

\section{References}

1 Sekar A, Bialas AR, de Rivera H, Davis A, Hammond TR, Kamitaki N, et al Schizophrenia risk from complex variation of complement component 4. Nature 2016; 530: 177-93.

2 Singh T, Kurki M, Curtis D, Purcell S, Cooks L, McRae J, et al. Rare SETD1A loss-of-function variants are associated with schizophrenia and developmental disorders. Nat Neurosci 2016; 19: 571-7.

3 Schizophrenia Working Group of the Psychiatric Genomics Consortium Biological insights from 108 schizophrenia-associated genetic loci. Nature 2014; 511: 421-7.

4 Rees E, Walters JTR, Georgieva L, Isles AR, Chambert KD, Richards AL, et al. Analysis of copy number variations at 15 schizophrenia-associated loci. Br J Psychiatry 2014; 204: 108-14.

5 St Clair D, Blackwood D, Muir W, Walker M, St Clair D, Muir W, et al. Association within a family of a balanced autosomal translocation with major mental illness. Lancet 1990; 336: 13-6.

6 Takata A, Xu B, Ionita-Laza I, Roos JL, Gogos JA, Karayiorgou M Loss-of-function variants in schizophrenia risk and SETD1A as a candidate susceptibility gene. Neuron 2014; 82: 773-80.

7 Dincer A, Gavin DP, Xu K, Zhang B, Dudley JT, schadt EE, et al. Deciphering H3K4me3 broad domains associated with gene-regulatory networks and conserved epigenomic landscapes in the human brain. Transl Psychiatry 2015; 5: e679.

8 The Network and Pathway Analysis Subgroup of the Psychiatric Genomics Consortium. Psychiatric genome-wide association study analyses implicate neuronal, immune and histone pathways. Nat Neurosci 2015; 18: 199-209.

9 Curtis D. Pathway analysis of whole exome sequence data provides further support for the involvement of histone modification in the aetiology of schizophrenia. Psychiatr Genet 2016; March 15 (Epub ahead of print). 